\title{
Dynamic Glass Transition and Fragility of Lithium Borate Binary Glass
}

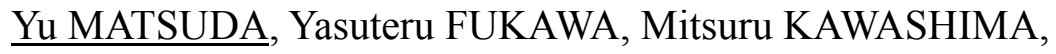 \\ Seiich MAMIYA and Seiji KOJIMA
}

Graduate School of Pure and Applied Sciences, University of Tsukuba, 1-1-1 Tennodai, Tsukuba, Ibaraki, 305-8573, Japan

email: s-matsuda@ims.tsukuba.ac.jp

\begin{abstract}
The compositional variations of the fragility of lithium borate binary glasses have been investigated over a wide composition range up to $64 \mathrm{~mol} \%$ of $\mathrm{Li}_{2} \mathrm{O}$. The frequency dependences of the dynamic heat capacity $C_{p}(\omega)$ above the dynamic glass transition temperature $T_{g}$ have been observed by Temperature-Modulated DSC. The fragility or steepness index $m$ has been determined. The composition dependence has been discussed on the basis of the fragility model proposed by Vilgis, and it has been revealed that the trends of the fragility and the fluctuation of the coordination number of boron atom have the correlation over a wide composition range. The result indicates the origin of the fragility relates to the degree of the fluctuation of the coordination number. The formation of the 4-coordinated boron atom plays the dominant role in the fragility of the borate glassy system.
\end{abstract}

Keywords: lithium borate glass; fragility; glass transition; relaxation time; steepness index; TMDSC; complex heat capacity 


\section{Introduction}

Lithium-doped borate glasses have the potential ability to be an excellent superionic conducting material [1-4]. Their physical properties, however, have not been sufficiently understood yet, since they show quite complicated behavior, termed as "borate anomaly" [5]. Even in the case of the lithium borate binary glasses, physical properties do not monotonically change with the increase of the alkali metal oxide content, but exhibit maxima or minima against their composition, for density [5], sound velocity [6, 7], non-Debye parameters [8-10] and boson peak [11]. Therefore, at the present stage, it is difficult to predict the physical properties of the ternary or more borate glasses containing lithium ion. Since it is important to firstly understand the nature of the binary system, we have paid attention to the lithium borate binary system, $x \mathrm{Li}_{2} \mathrm{O} \cdot(100-\mathrm{x}) \mathrm{B}_{2} \mathrm{O}_{3}$ where $x$ is the molar concentration in $\mathrm{Li}_{2} \mathrm{O}$, as a fundamental system of lithium-doped borate glasses.

In this paper, the temperature dependences of the structural relaxational process in supercooled liquid, called $\alpha$-process, have been investigated over a wide range of composition of $\mathrm{Li}_{2} \mathrm{O}$ above the glass transition temperature. We have discussed the origin of the compositional variations of the temperature evolution of the structural relaxation time near the glass transition temperature $T_{g}$, frequently termed as "fragility [12]" in glass science.

\section{Fragility and Temperature-Modulated DSC}

The concept of the "fragility" in glass-forming materials was introduced by Angell in order to classify the temperature variations of the $\alpha$-relaxation process [12]. The classification is based on an Arrhenius plot of the temperature dependence of viscosity or $\alpha$-relaxation time $\tau$ above $T_{g}$, in which the horizontal axis is normalized by $T_{g}$ of a material (see Figure 1). This figure is usually called Angell plot. A "strong" liquid exhibits the Arrhenius temperature dependence, while a "fragile" liquid shows a notable deviation from Arrhenius behavior, 
indicating the rapid temperature evolution of $\tau$ near $T_{g}$. The origin of the fragility is one of the central topics in glass science [13]. However, it is not fully understood yet. In other words, it is not clear why the degree of the deviation from the Arrhenius law near $T_{g}$ is different in various glass formers. Moreover, recently the relationship between the fragility above $T_{g}$ and the elastic property in the glassy state has been hotly debated [14-15]. The findings of the origin will give new insights into the glass transition dynamics and fundamentals of the borate system.

Lee et al. investigated the fragility of the some borate glass system: $A g I-A g g_{2} O-B_{2} O_{3}$ [16] and $\mathrm{Na}_{2} \mathrm{O}-\mathrm{B}_{2} \mathrm{O}_{3}$ [17]. They discussed the compositional variations of the fragility determined by the viscosity measurement on the basis of the Phillips's idea and suggested the relationship between the fragility and average coordination number [17].

The previous study by Chryssikos et al. revealed that the fragility of the lithium borate binary system drastically varies by the addition of $\mathrm{Li}_{2} \mathrm{O}$ to $\mathrm{B}_{2} \mathrm{O}_{3}$ [18]. However, the composition range studied was limited up to about $\mathrm{x}=25 \mathrm{~mol} \%$ because of the poor glass-forming ability and high ionic conductivity of the high composition lithium borate glasses. These problems obstruct the viscosity measurement and dielectric spectroscopy above $T_{g}$ for the high compositions. The origin of the fragility of lithium borate binary glass is not fully understood yet.

In this context, we have already shown that the Temperature-Modulated DSC (TMDSC) technique can investigate the fragility by observing the frequency-dependent dynamic heat capacity near $T_{g}[19]$. The method to determine the fragility qualitatively by TMDSC has been discussed in [19]. The present paper is the sequel of [19], and the purpose is to investigate the composition dependence of the fragility over a wide composition range, i. e. up to $x=64 \mathrm{~mol} \%$. The origin has been discussed based on the structural changes of the borate network. 


\section{Experimental}

The detail description for the sample preparation and TMDSC has been written in [6, 8-10]. The chemical formula is denoted by $x \mathrm{Li}_{2} \mathrm{O} \cdot(100-\mathrm{x}) \mathrm{B}_{2} \mathrm{O}_{3}$, where $x$ is the molar concentration in $\mathrm{Li}_{2} \mathrm{O}$. In order to investigate the inherent nature of the binary system, the high homogeneous samples were prepared by the "solution method" [6, 10]. Analytical reagent grade $\mathrm{LiOH} \cdot \mathrm{H}_{2} \mathrm{O}$ and $\mathrm{H}_{3} \mathrm{BO}_{3}$ were used as the starting materials without further purification. These starting materials are initially made to react in an aqueous solution in order to achieve high homogeneity. After the complete evaporation of water in a dry box, a chemically synthesized powder was obtained. The powder was fused in a Pt crucible at about 950 to $1300{ }^{\circ} \mathrm{C}$ depending on the composition, for 1.5 hours. The glasses were obtained by a plate quenching. The quenched samples were used as is without any annealing because of the crystallization.

In TMDSC, a small sinusoidal temperature heating rate is superimposed on a linear heating rate used in a conventional DSC. Then, the modulated heat flow can be obtained as a response to the modulated heating rate. An absolute value of a dynamic heat capacity can be obtained using eq. (1)

$$
\left|C_{p}^{*}(\omega)\right|=\frac{A_{H F}}{A_{q}},
$$

where $A_{q}$ is the amplitude of the modulation in the heating rate (external force) and $A_{H F}$ is the amplitude of the modulation in the observed heat flow (response). By using phase angle $\theta$, $\left|C_{p}^{*}(\omega)\right|$ can be separated into real $C_{p}{ }^{\prime}(\omega)$ and imaginary $C_{p}{ }^{\prime \prime}(\omega)$ parts using eqs. (2) and (3),

$$
\begin{aligned}
& C_{p}^{\prime}(\omega)=\left|C_{p}^{*}(\omega)\right| \cos \theta, \\
& C_{p}^{\prime \prime}(\omega)=\left|C_{p}^{*}(\omega)\right| \sin \theta .
\end{aligned}
$$

The dynamic heat capacity can be explained by the linear response theory and 
fluctuation-dissipation theorem [20 - 22]. It is noted that the dynamic heat capacity is a frequency-dependent, and one can access the temperature-dependence of $\tau$ by the observation of its frequency dependence.

The experimental conditions for the TMDSC measurements are as follows. The TMDSC (DSC2920 and Q200, TA Instruments) were used. The temperature and enthalpy were calibrated by the heat of fusion of pure indium. The values of $\left|C_{p}^{*}(\omega)\right|$ were calibrated using the sapphire disk provided by TA Instrument in the studied temperature range. A powdered sample in an aluminum pan was heated with the temperature profile; underlying heating rate is $0.5-1.0^{\circ} \mathrm{C} / \mathrm{min}$, modulation period $P$ is $20-200 \mathrm{sec}$ and the temperature amplitude is $\pm 0.5-1.0^{\circ} \mathrm{C}$.

The method for the phase angle correction used in this paper was the same as that in previous paper [17], in which it has been shown that the proper phase correction is important. Basically, the method proposed by Weyer was used [23]. In shorter period experiments, an additional correction was required because a baseline of raw phase angles was not flat, while the phase angle at the longer period was flat. In such a case, a shape of the baseline was checked by a sapphire or empty run measurements. After the subtraction of the baseline from the raw phase angle, the Weyer's method was applied.

\section{Results and Discussion}

The temperature dependences of $\left|C_{p}^{*}(\omega)\right|$ and raw phase angle have been presented in $[9-10,19]$. Here, figure 2 shows the frequency dependence of the real $C_{p}{ }^{\prime}(\omega)$ and imaginary $C_{p}{ }^{\prime \prime}(\omega)$ parts for $x=10 \mathrm{~mol} \%$. The $C_{p}{ }^{\prime}(\omega)$ shows the step-wise change, and $C_{p}{ }^{\prime \prime}(\omega)$ has broad peak around the peak temperature $T_{\max }^{\omega}$. These are the characteristic feature of the relaxation phenomena related to the dynamic glass transition. 
The change of the frequency of the temperature modulation in TMDSC enables us to perform the heat capacity spectroscopy. $T_{\max }^{\omega}$ shifts to higher temperature as the modulation period $P$ becomes shorter. In TMDSC experiments, the period or frequency of the modulation is fixed, whereas the relaxation times $\tau$ of the $\alpha$-process drastically varies with the change of temperature. The characteristic $\tau$ is the most probable at $T_{\max }^{\omega}$ with the constant $P$. Therefore, the temperature dependence of $\tau\left(T_{\max }^{\omega}\right)$ can be given by,

$$
\tau=\frac{P}{2 \pi}=\frac{1}{\omega}
$$

where $\omega$ is the angular frequency of the temperature modulation.

Figure 3 a) shows the Arrhenius plots for $x=10$ and 42, in which the common logarithm of $\tau(T)$ is plotted against reciprocal temperature. A value of $T_{g}$ is defined as the temperature when $\tau$ becomes $100 \mathrm{sec}, i . e . \log \tau=2$. In the present study, the value of $T_{g}$ has been determined by the extrapolation from the experiment data, as shown by Fig. 3 a). The values of $T_{g}$ are about $643 \mathrm{~K}(\mathrm{x}=10)$ and $736 \mathrm{~K}(\mathrm{x}=40)$, respectively. These values are comparable to those obtained DSC measurement in which $T_{g}$ is defined as a onset temperature of a glass transition in a heating run $[5,6]$.

Figure $3 \mathrm{~b}$ ) shows the Angell plot where the reciprocal temperature is normalized by $T_{g}$. It is clear that the fragility of lithium borate glasses increase with the increase of the $\mathrm{Li}_{2} \mathrm{O}$ content. In order to evaluate the fragility quantitatively, the fragility or steepness index $m$ defined using equation (5) [12] is frequently used ;

$$
m=\lim _{T \rightarrow T_{g}}\left|\frac{d \log \tau}{d\left(T_{g} / T\right)}\right|,
$$


which is proportional to an apparent activation energy at $T=T_{g}$ for viscous flow. Although $\tau(T)$ is usually expressed by the Vogel-Fulcher (VF) law in a wide temperature range, we assume that the present data obtained by TMDSC are described by a linear dependence near $T_{g}$. This assumption is valid at least in this study because the data cover only a very limited range of the $T_{g} / T$ scale near $T_{g}\left(0.96<T_{g} / T<1\right)$. Therefore, the value of $m$ can be easily determined as the value of the slope, shown in Fig. 3 b). The fragility or steepness index is a convenient measure of a degree of the fragility. As was discussed in the previous paper [19], the value of $m$ for $\mathrm{x}=10$ is very close to that obtained by the viscosity measurement, when the proper phase angle correction was applied.

The compositional variations of $m$ over a wide composition range up $\mathrm{x}=64 \mathrm{~mol} \%$ have been presented in Fig. 4 a). The closed circles denote the experimental values by TMDSC, and the open circles denote the reference values summarized by Chryssikos et al. from the viscosity data [18]. Here, the composition range studied has been extended up to $64 \mathrm{~mol} \%$. The fragility slightly increase in $0<\mathrm{x}<10$, then shows the dramatic change step-wisely in 10 $<\mathrm{x}<30$, and then, has a very broad maximum. Surprisingly, the extreme high composition glasses seem to have the strong character in terms of the fragility.

To understand this composition dependence, the theoretical fragility model proposed by Vilgis is useful [24-25]. The summary of the model is that a degree of the fragility relates to a degree of the fluctuation of the coordination number of a key element. For instance, a vitreous silica $\mathrm{SiO}_{2}$ belongs to the "strong" since it has a fixed covalent bonding of $\mathrm{Si}$ atom, which is 4-coordinated to oxygen atoms. On the other hand, o-therphenyl (OTP), one of well-known molecular glass-former, belongs to the typical "fragile" one since its coordination number fluctuates from 11 to 16 [25], indicating that the molecules strongly interact with each other cooperatively. 
In the case of the lithium borate glasses, it is known that the coordination number of a boron atom changes from 3 to 4 with the $\mathrm{Li}_{2} \mathrm{O}$ content [26-29]. A pure $\mathrm{B}_{2} \mathrm{O}_{3}$ glass is constructed from the random network of the 6-membered boroxol rings consisted of $\mathrm{BØ}_{3}$ triangular planer units, where all boron atoms are 3-coordinated with bridging oxygen atoms, $\varnothing$. The addition of $\mathrm{Li}_{2} \mathrm{O}$ to $\mathrm{B}_{2} \mathrm{O}_{3}$ glass causes the formation of $\mathrm{B}_{4}$ tetrahedral groups, where part of boron atoms is 4-coordinated (see Fig. 5). The composition dependence of the fraction of the 4-coorinated boron atom, $N_{4}(x)$, has been determined extensively by NMR [26-27] and vibrational spectroscopy [28-29]. Therefore, the fluctuation, $F$, of the coordination number of boron atom can be calculated using eqs. (6) on the basis of the discrete probability theory:

$$
\begin{aligned}
& F(x)=(3-\bar{Z}(x))^{2} \cdot N_{3}(x)+(4-\bar{Z}(x))^{2} \cdot N_{4}(x), \\
& \bar{Z}(x)=3 \cdot N_{3}(x)+4 \cdot N_{4}(x), \\
& N_{3}(x)+N_{4}(x)=1,
\end{aligned}
$$

where $\bar{Z}(x)$ is a mean coordination number of boron atom and $N_{3}(x)$ or $N_{4}(x)$ is the fraction of 3 or 4-coodinated boron atom, respectively. The values for $N_{4}(x)$ are taken from Ref. [27].

The composition dependence of the fluctuation is shown in Fig. 4 b). In comparison of Fig. 4 a) with Fig. 4 b), it is clear that behavior of each trend is roughly similar. This result indicates that the origin of the fragility surely relates to the degree of the fluctuation of the coordination number of a system. In other words, the present work has provided the experimental evidence for the prediction by Vilgis.

S. $-\mathrm{K}$. Lee et al. investigated the fragility of sodium silicate glasses, $\mathrm{Na}_{2} \mathrm{O}-\mathrm{SiO}_{2}$, on the basis of the viscosity and thermal properties, and found that the fragility of $\mathrm{Na}_{2} \mathrm{O}-\mathrm{SiO}_{2}$ glasses monotonically increases as the $\mathrm{Na}_{2} \mathrm{O}$ content increase [30]. In such a case, the coordination number of silicon atom is constant to be four but non-bridging oxygen atoms increased with an increase in the alkali oxide contents. Aniya has also proposed the extended model of the fragility, in which not only the coordination number but also the bond strength are considered, and it has been shown that the degrees of both fluctuation relate to the fragility [31]. This 
model may be applicable to explain the fragility of both alkali silicate and borate glasses. In the alkali silicate glasses, the atomic units, $\mathrm{SiO}_{4}$ tetrahedra, change from $Q_{4}$ to $Q_{0}$ as alkali oxide content increases, where the subscript denotes the number of bridging oxygens per silicon tetrahedron. It is known that $Q_{n}(\mathrm{n}=0,1,2,3,4)$ systematically varies with the increase of alkali oxide such as $\mathrm{Q}_{4} \rightarrow \mathrm{Q}_{3} \rightarrow \mathrm{Q}_{2} \rightarrow \mathrm{Q}_{1} \rightarrow \mathrm{Q}_{0}$. In contrast to silicate glasses, the situation of borate glasses is rather complicated since the several structural units exist. Thus, in this paper, we have firstly considered the coordination change of boron atom, and revealed that the fluctuation of coordination number of boron atom correlates to the fragility of the system. This result indicates that in the case of binary lithium borate glasses, the coordination change of boron atom is more important factor into the origin of the fragility than that of oxygen atom.

Ikeda and Aniya have been provided the new method to analyze the fragility [32]. In this model, if the knowledge for the fluctuation of the coordination number is known, the parameters relating to the fluctuation of the bond strength can be determined. This model can give new additional insights into the origin of the fragility. Such analysis by this model on the basis of the coordination change of both boron and oxygen atoms is our future work, and the results will be shown in a next paper.

In summary, the frequency dependences of the dynamic heat capacity $C_{p}(\omega)$ of the lithium borate binary system have been observed above the dynamic glass transition temperature $T_{g}$ by the Temperature-Modulated DSC technique. The fragility or steepness index $m$ has been determined over a wide range of compositions up to $\mathrm{x}=62 \mathrm{~mol} \%$, by the temperature dependences of the structural relaxation time in the vicinity of the $T_{g}$. The composition dependence has been discussed based on the Vilgis model, and it has been revealed that the trend of the fragility and the fluctuation of the coordination number of boron atom are similar over a wide composition range. The results indicate the origin of the fragility 
relates to the degree of the fluctuation of the coordination number. In the case of the borate glasses, the fragility can strongly correlate to the formation of the 4-coordinated boron atom.

\section{Acknowledgements}

The authors gratefully acknowledge helpful discussion with Prof. Masao Kodama, Department of Applied Chemistry, Sojo Univ., for the sample preparation and characterization. (Y. M) is also thankful for the JSPS Research Fellowship for Young Scientist. This research was partially supported by MEXT, the Grant-in-Aids for JSPS Fellow 19.574. 


\section{References}

[1] B. V. R. Chowdari, Z. Rong, Solid State Ionics 90 (1996) 151.

[2] T. Matsuo, M. Shibasaki, T. Katsumata, Solid State Ionics 154-155 (2002) 759.

[3] C. H. Lee, H. J. Sohn, M. G. Kim, Solid State Ionics 176 (2005) 1237.

[4] V. C. Veeranna Growda, R. V. Anavekar, Solid State Ionics 176 (2005) 1393.

[5] M. Affatigato, S. Feller, E. J. Khaw, D. Feil, B. Teoh, O. Mathews, Phys. Chem. Glasses 31 (1990) 19.

[6] M. Kodama, T. Matsushita, S. Kojima, Jpn. J. Appl. Phys. 34 (1994) 2570.

[7] Y. Fukawa, Y. Matsuda, Y. Ike, M. Kodama, S. Kojima, Jpn. J. Appl. Phys., accepted

[8] Y. Matsuda, Y. Fukawa, Y. Ike, M. Kodama, S. Kohima. J. Phys. Soc. Jpn., accepted

[9] Y. Matsuda, C. Matsui, Y. Ike, M. Kodama, S. Kojima, J. Therm. Anal. Cal. 85 (2006) 725.

[10] Y. Matsuda, Y. Fukawa, C. Matsui, Y. Ike, M. Kodama, S. Kojima, Fluid. Phase Eq. 256 (2007) 127.

[11] S. Kojima, V. N. Novikov, M. Kodama, J. Chem. Phys. 113 (2000) 6344.

[12] C. A. Angell, J. Non-Cryst. Solid 131-133 (1991) 13.

[13] C. A. Angell, K. L. Ngai, G. B. McKenna, P. F. McMillan, S. W. Martin, J. Appl. Phys. 88 (2000) 3113.

[14] V. N. Novikov, A. P. Sokolov, Nature 431 (2004) 961

[15] S. N. Yannopoulus, G. P. Johari, Nature 442 (2006) E7

[16] S. -K. Lee, M. Tatsumisago and T. Minami, Phys. Chem. Glasses 38 (1997) 144.

[17] S. -K. Lee, M. Tatsumisago and T. Minami, J. Ceram. Soc. Jpn 103 (1995) 398.

[18] G. D. Chyssikos, J. A. Duffy, J. M. Hutchinson, M. D. Ingram, E. I. Kamitsos, A. J. Pappin, J. Non-Cryst. Solid 172-174 (1994) 378.

[19] Y. Matsuda, Y. Fukawa, M. Kawashima, S. Kojima, AIP Conference Proc. accepted, (Proc. of the $5^{\text {th }}$ International Workshop on Complex System).

[20] N. O. Birge, Phys. Rev. B 34 (1986) 1631.

[21] S. Kojima, T. Kashiwada, S. Nakahira, High Temp.-High Press. 23 (1991) 701.

[22] S. Kojima, T. Koketsu, E. Takahashi, M. Kanayasu, Fluid Phase Eq. 88 (1993) 209.

[23] S. Weyer, A. Hensel, C. Schick, Thermochim Acta 304/305 (1997) 267.

[24] T. A. Vilgis, J. Phys.:Condens. Matter 2 (1990) 3667.

[25] T. A. Vilgis, Phys. Rev. B 47 (1993) 2882.

[26] P. J. Bray, J. G. ÓKeefe, Phys. Chem. Glasses 4 (1963) 37. 
[27] G. E. Jellison, S. A. Feller, P. J. Bray, Phys. Chem. Glasses 19 (1978) 52.

[28] E. I. Kamitsos, M. A. Karakassides, G. D. Chryssikos, Phys. Chem. Glasses 28 (1987) 203.

[29] E. I. Kamitsos, A. P. Patsis and M. A. Karakassides and G. D. Chryssikos, J. Non-Cryst. Solids 126 (1990) 52.

[30] S. -K. Lee, M. Tatsumisago and T. Minami, J. Ceram. Soc. Jpn. 101 (1993) 1018.

[31] M. Aniya, J. Therm. Anal. 69 (2002) 971.

[32] M. Ikeda and M. Aniya, in this issue. 


\section{Figure captions}

Figure 1. The schematic diagram for the fragility (Angell plot). The common logarithm of $\tau$ is plotted against the $T_{g}$-scaled reciprocal temperature. (Color online)

Figure 2. The temperature and frequency dependence of the real and imaginary parts for $\mathrm{x}=$ $10 \mathrm{~mol} \%$. The peak temperatures $T_{x}$ shift to higher as the period of the temperature modulation becomes shorter. (Color online)

Figure 3. a) Arrhenius plot ( $\log \tau$ vs. $1 / T$ ) for the structural relaxation times $\tau$ for $\mathrm{x}=10$ and $42 \mathrm{~mol} \%$, where $T_{g}$ is defined as the temperature when $\tau=100 \mathrm{sec}$. The DSC 2920 was used for the measurement of $x=10$. The Q200 was used for $x=42$. (Color online). $b$ ) Angell plot ( $\log \tau$ vs. $\left.T_{g} / T\right) . T_{g}$ is determined by the extrapolation for the experimental data in the Arrhenius plot. The slop of the straight line is equivalent to that of the fragility index $m$. (Color online)

Figure 4. a) The composition dependence of the fragility index $m$ of the lithium borate glasses. Open circles denote the reference values [18]. Closed circles denote the present results by TMDSC. The solid line is a guide for eyes. (Color online). b) The composition dependence of the fluctuation of the coordination number of boron atom calculated by NMR data [27]. (Color online)

Figure 5. The schematic diagram of the borate structural units for 3-coordinated and 4-coordinated boron. Open circles denote oxygen atoms. Closed circles denote boron atoms. (Color online) 


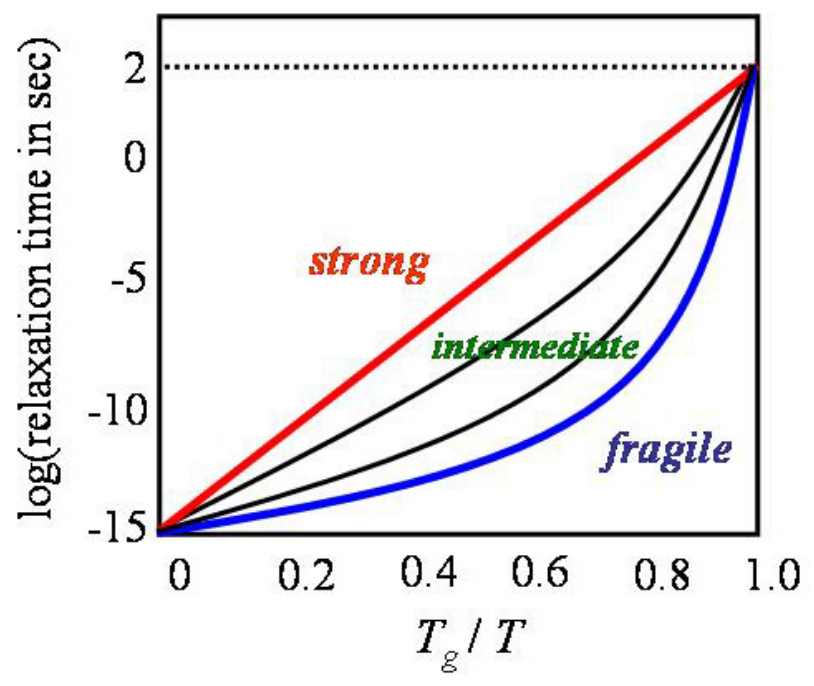

Figure 1

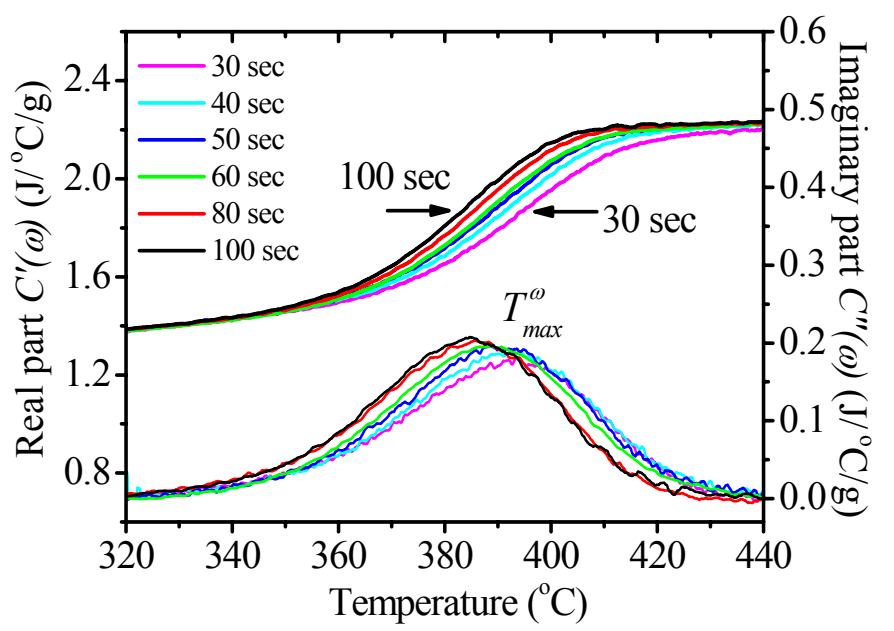

Figure 2 

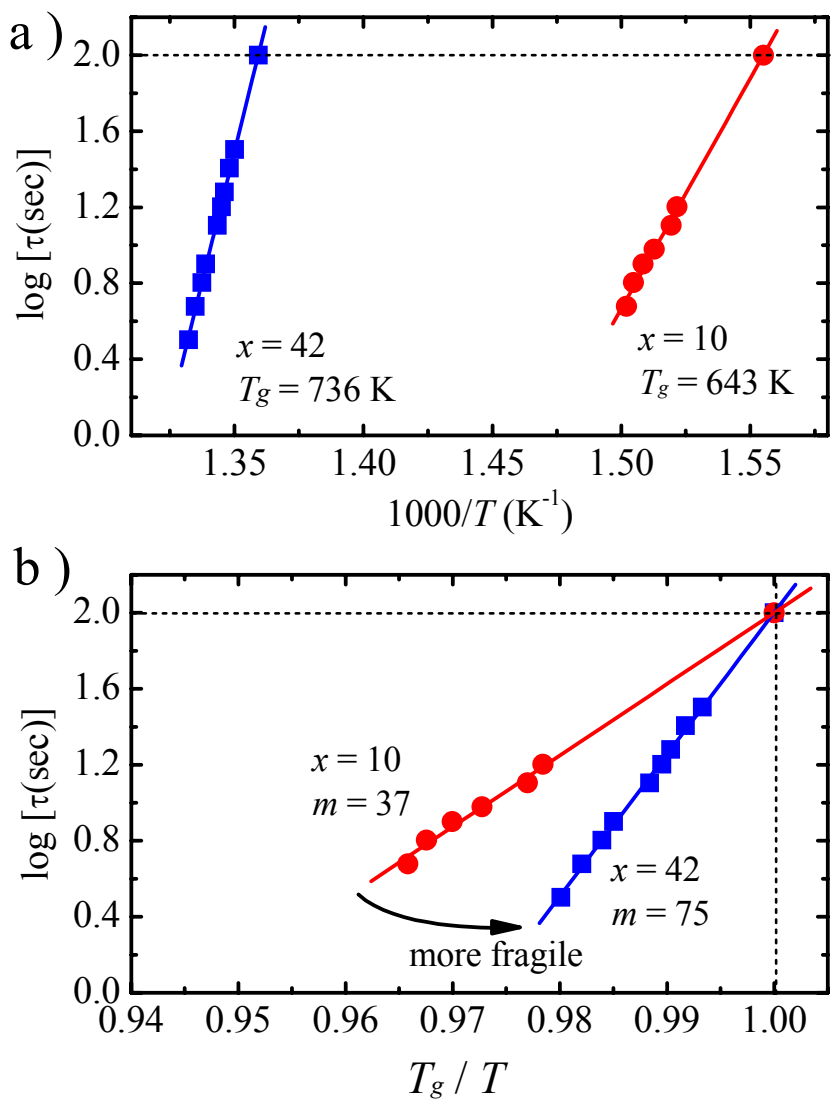

Figure 3 

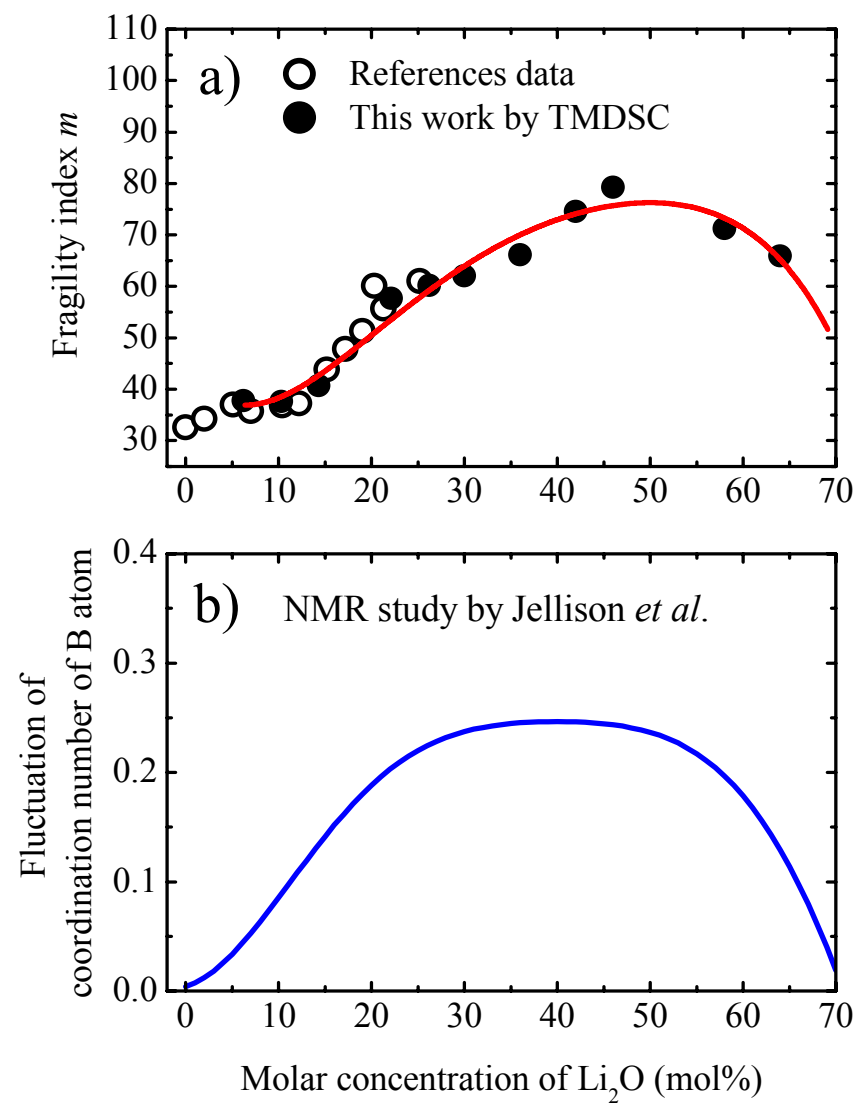

\section{Figure 4}




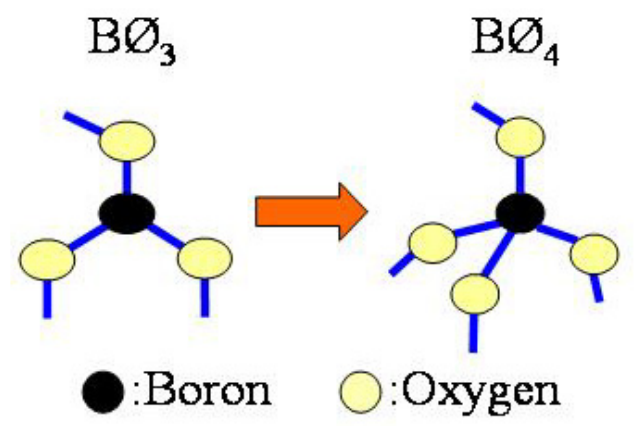

Figure 5 